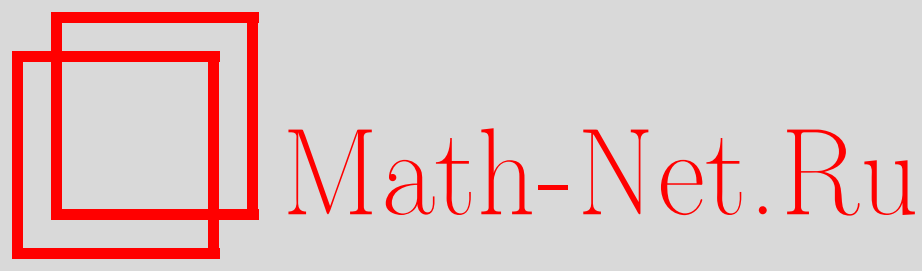

Н. Н. Боголюбов (мл.), А. В. Глущенко, М. Ю. Ковалевский, Квазисредние и вырожденные квантовые состояния равновесия магнитных систем с $S U(3)$-симметрией обменного взаимодействия, ТMФ, 2018, том 195, номер 2, 240-255

DOI: https://doi.org/10.4213/tmf9397

Использование Общероссийского математического портала Math-Net.Ru подразумевает, что вы прочитали и согласны с пользовательским соглашением http://www. mathnet.ru/rus/agreement

Параметры загрузки:

IP : 52.23 .180 .231

26 апреля 2023 г., $17: 15: 52$

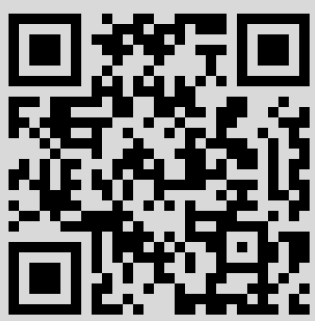


Том 195, № 2

май, 2018

(C) 2018 г.

Н. Н. Боголюбов (мл.)*, А. В. Глущенко ${ }^{\dagger}$, М. Ю. Ковалевский ${ }^{\dagger}$

\title{
КВАЗИСРЕДНИЕ И ВЫРОЖДЕННЫЕ КВАНТОВЫЕ СОСТОЯНИЯ РАВНОВЕСИЯ МАГНИТНЫХ СИСТЕМ С $S U(3)$-СИММЕТРИЕЙ ОБМЕННОГО ВЗАИМОДЕЙСТВИЯ
}

\begin{abstract}
Рассмотрены магнитные системы с $S U(3)$-симметрией обменного взаимодействия. Для вырожденных состояний равновесия с нарушенной магнитной и фазовой симметриями с использованием представления об остаточной симметрии сформулированы уравнения классификации для параметра порядка. На их основе получен явный вид равновесных значений параметров порядка спинового нематика и антиферромагнетика в общем виде. Выяснены условия существования шести типов сверхтекучих состояний равновесия для параметра порядка, описывающего парный бозе-конденсат. Изучены неоднородные состояния равновесия и получена явная зависимость магнитных параметров порядка от координаты.
\end{abstract}

Ключевые слова: равновесие, параметр порядка, спин, унитарная симметрия, уравнения классификации.

DOI: https://doi.org/10.4213/tmf9397

\section{1. ВВЕДЕНИЕ}

Высокоспиновые магнетики в настоящее время являются объектом, вызывающим большой научный интерес. В этих многочастичных системах возможны необычные типы магнитного упорядочения, к которым, в частности, относятся квадрупольные, ферроквадрупольные, антиферроквадрупольные состояния, а также состояния спиновых нематиков и антиферронематиков [1]-[6]. Недавнее прямое экспериментальное подтверждение существования спинового нематика [7] стимулировало дальнейшее развитие интереса к этой области исследований. В работах [8]-[10] изучены

* Математический институт им. В. А. Стеклова Российской академии наук, Москва, Россия. E-mail: nikolai_bogolubov@hotmail.com

${ }^{\dagger}$ Национальный научный центр "Харьковский физико-технический институт”, Харьков, Украина. E-mail: gluschenko.ant@gmail.com, mikov51@mail.ru 
состояния равновесия, в которых спонтанно нарушены фазовая и магнитная симметрии. На основе модельного вида обменной энергии как функции спинорного параметра порядка исследованы сверхтекучие состояния равновесия и рассмотрены вопросы сосуществования ферромагнетизма и явления бозе-конденсации. Аналогичная задача об основном состоянии бозе-систем со спином $s=1$, где существенным является представление об остаточной симметрии вырожденного состояния равновесия [11], [12], проанализирована в теоретико-групповом подходе [13]-[15], где обменное взаимодействие обладает $S O(3)$-симметрией. В статьях [16]-[18] рассмотрены физические особенности конденсированных состояний магнетиков со спином $s=1$ и $S U(3)$-симметрией обменного взаимодействия.

Нами исследовано влияние унитарной $S U(3)$-симметрии обменного взаимодействия на возможные магнитные и сверхтекучие состояния равновесия в бозе-системах со спином $s=1$. С использованием концепции квазисредних [19], [20] в таком подходе ранее были классифицированы однородные и неоднородные состояния равновесия сверхтекучего ${ }^{3} \mathrm{He}[21]$, [22], а также жидкокристаллические и магнитные состояния со спином $s=1 / 2$ [23]. Обобщение указанного подхода на магнетики со спином $s=1$ дано в работе [24]. Представление о генераторе остаточной симметрии совместно с трансформационными свойствами параметра порядка приводят к уравнениям классификации параметра порядка. Анализ решений этих уравнений позволяет установить структуру анизотропии параметра порядка в терминах параметров генератора этой симметрии.

Структура статьи имеет следующий вид. В разделе 2 рассмотрены нормальные состояния равновесия изучаемых магнитных систем и сформулированы их свойства симметрии. В разделе 3 дан анализ состояний равновесия со спонтанно нарушенной магнитной симметрией и фазовой симметрией. Сформулированы в общем виде уравнения классификации для параметра порядка. В разделе 4 изучен антиферромагнитный параметр порядка и параметр порядка спинового нематика. Получен явный вид этих параметров порядка в состоянии равновесия в общем случае. В разделе 5 рассмотрены возможные сверхтекучие состояния с билинейным по бозе-оператору параметром порядка, который отвечает парному конденсату и учитывает магнитные степени свободы. Дан анализ сверхтекучих состояний равновесия с таким параметром порядка и выяснен характер их магнитной анизотропии в состоянии равновесия. Раздел 6 посвящен изучению неоднородных магнитных состояний равновесия. Сформулировано условие остаточной пространственной симметрии и найдена координатная зависимость параметров порядка в состоянии равновесия.

\section{2. НОРМАЛЬНЫЕ СОСТОЯНИЯ РАВНОВЕСИЯ И ИХ СВОЙСТВА СИММЕТРИИ}

Нормальные состояния равновесия магнетиков со спином $s=1$ и унитарной $S U(3)$-симметрией обменного взаимодействия описываются статистическим оператором Гиббса, который имеет вид

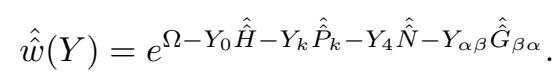

Аддитивные интегралы движения $\hat{\hat{\gamma}}_{a}=\left(\hat{\hat{H}}, \hat{\hat{P}}_{k}, \hat{\hat{N}}, \hat{\hat{G}}_{\alpha \beta}\right)$, где $a=0, k, 4, \alpha \beta$, включают операторы Гамильтона $\hat{\hat{H}}$, импульса $\hat{\hat{P}}_{k}$, числа частиц $\hat{\hat{N}}$ и магнитный интеграл 
движения $\hat{\hat{G}}_{\alpha \beta}$ в базисе Вейля [25]

$$
\hat{\hat{G}}_{\alpha \beta}=\int d^{3} x\left(\hat{\hat{\psi}}_{\alpha}^{+}(\mathbf{x}) \hat{\hat{\psi}}_{\beta}(\mathbf{x})-\delta_{\alpha \beta} \hat{\hat{\psi}}_{\gamma}^{+}(\mathbf{x}) \frac{\hat{\hat{\psi}}_{\gamma}(\mathbf{x})}{3}\right)=\int d^{3} x \hat{\hat{g}}_{\alpha \beta}(\mathbf{x}),
$$

который представлен в терминах полевых бозе-операторов, $\alpha, \beta=x, y, z$. Этот оператор имеет физический смысл генератора $S U(3)$-симметрии и удовлетворяет соотношениям $\hat{\hat{G}}_{\alpha \beta}=\hat{\hat{G}}_{\beta \alpha}^{+}, \hat{\hat{G}}_{\alpha \alpha}=0$. Его связь с эрмитовыми операторами спина и квадрупольной матрицей магнитной системы задана равенством $\hat{\hat{G}}_{\alpha \beta} \equiv \hat{\hat{Q}}_{\alpha \beta}+i \varepsilon_{\alpha \beta \gamma} \hat{\hat{S}}_{\gamma} / 2$. Термодинамические силы $Y_{a}$, входящие в распределение Гиббса, сопряжены аддитивным интегралам движения. Ввиду эрмитовости статистического оператора справедливо соотношение $Y_{\alpha \beta}=Y_{\beta \alpha}^{+}$. Вместо этой матрицы далее мы используем действительную векторную величину $Y_{a}=i \varepsilon_{\alpha \beta \gamma} Y_{\beta \gamma} / 2$ и симметричную матрицу $Y_{\alpha \beta}^{(s)}=\left(Y_{\alpha \beta}+Y_{\beta \alpha}\right) / 2$, так что $Y_{\alpha \beta} \hat{\hat{G}}_{\beta \alpha}=Y_{\alpha} \hat{\hat{S}}_{\alpha}+Y_{\alpha \beta}^{(s)} \hat{\hat{Q}}_{\alpha \beta}$. Ввиду бесследовости оператора (2) полагаем $Y_{\alpha \alpha}^{(s)}=0$.

Сформулируем свойства симметрии нормальных состояний равновесия рассматриваемых магнетиков. К ним относятся инвариантность статистического оператора (1) относительно фазовых преобразований, а также пространственная однородность и изотропия состояния равновесия

$$
[\hat{\hat{w}}, \hat{\hat{N}}]=0, \quad\left[\hat{\hat{w}}, \hat{\hat{P}}_{k}\right]=0, \quad\left[\hat{\hat{w}}, \hat{\hat{L}}_{i}(\mathbf{Y})\right]=0 .
$$

Здесь обобщенный оператор орбитального момента определен равенствами

$$
\hat{\hat{L}}_{i}(\mathbf{Y}) \equiv \hat{\hat{L}}_{i}+L_{i}(\mathbf{Y}), \quad \hat{\hat{L}}_{i}=\varepsilon_{i k l} \int d^{3} x x_{k} \hat{\hat{\pi}}_{l}(\mathbf{x}), \quad L_{i}(\mathbf{Y}) \equiv-i \varepsilon_{i k l} Y_{k} \frac{\partial}{\partial Y_{l}},
$$

где $\hat{\hat{\pi}}(\mathbf{x})$ - оператор плотности импульса. Наряду с соотношениями (3) имеет место условие симметрии магнитной системы в спиновом пространстве

$$
\left[\hat{\hat{w}}, \hat{\hat{G}}_{\alpha \beta}(\hat{Y})\right]=0
$$

Обобщенный генератор $S U(3)$-симметрии определяется равенствами

$$
\hat{\hat{G}}_{\alpha \beta}(\hat{Y})=\hat{\hat{G}}_{\alpha \beta}+G_{\alpha \beta}(\hat{Y}), \quad G_{\alpha \beta}(\hat{Y})=Y_{\alpha \nu} \frac{\partial}{\partial Y_{\beta \nu}}-Y_{\nu \beta} \frac{\partial}{\partial Y_{\nu \alpha}} .
$$

В дальнейшем нам удобно представить свойство магнитной симметрии в терминах действительных магнитных термодинамических сил

$$
\hat{\hat{G}}_{\alpha \beta}(\hat{Y}) \rightarrow \hat{\hat{G}}_{\alpha \beta}(\hat{\mathbf{Y}})=\hat{\hat{G}}_{\alpha \beta}+G_{\alpha \beta}(\hat{\mathbf{Y}}) \equiv \hat{\hat{Q}}_{\alpha \beta}(\hat{\mathbf{Y}})+i \varepsilon_{\alpha \beta \gamma} \frac{\hat{\hat{S}}_{\gamma}(\hat{\mathbf{Y}})}{2} .
$$

Здесь $(\hat{\mathbf{Y}}) \equiv\left(\mathbf{Y}, \hat{Y}^{(s)}\right)$ - действительные магнитные термодинамические параметры. Обобщенные операторы спина и квадрупольной матрицы определяются соотношениями

$$
\begin{gathered}
\hat{\hat{Q}}_{\alpha \beta}(\hat{\mathbf{Y}})=\hat{\hat{Q}}_{\alpha \beta}+Q_{\alpha \beta}(\hat{\mathbf{Y}}), \quad \hat{\hat{S}}_{\gamma}(\hat{\mathbf{Y}})=\hat{\hat{S}}_{\gamma}+S_{\gamma}(\hat{\mathbf{Y}}) \\
Q_{\alpha \beta}(\hat{\mathbf{Y}}) \equiv i\left(Y_{\alpha \lambda}^{(s)} \varepsilon_{\lambda \beta \gamma}+Y_{\beta \lambda}^{(s)} \varepsilon_{\lambda \alpha \gamma}\right)+2 i Y_{\sigma}\left(\varepsilon_{\sigma \beta \lambda} \frac{\partial}{\partial Y_{\lambda \alpha}^{(s)}}+\varepsilon_{\sigma \alpha \lambda} \frac{\partial}{\partial Y_{\lambda \beta}^{(s)}}\right) \\
S_{\gamma}(\hat{\mathbf{Y}}) \equiv-i \varepsilon_{\alpha \beta \gamma}\left(Y_{\alpha} \frac{\partial}{\partial Y_{\beta}}+2 Y_{\alpha \lambda}^{(s)} \frac{\partial}{\partial Y_{\beta \lambda}^{(s)}}\right)
\end{gathered}
$$


Из явного вида этих операторов получаем квантовую алгебру

$$
\begin{aligned}
i\left[\hat{\hat{S}}_{\alpha}(\hat{\mathbf{Y}}), \hat{\hat{S}}_{\beta}(\hat{\mathbf{Y}})\right] & \equiv-\varepsilon_{\alpha \beta \gamma} \hat{\hat{S}}_{\gamma}(\hat{\mathbf{Y}}), \\
i\left[\hat{\hat{S}}_{\alpha}(\hat{\mathbf{Y}}), \hat{\hat{Q}}_{\beta \gamma}(\hat{\mathbf{Y}})\right] & \equiv-\varepsilon_{\alpha \gamma \sigma} \hat{\hat{Q}}_{\sigma \beta}(\hat{\mathbf{Y}})-\varepsilon_{\alpha \beta \sigma} \hat{\hat{Q}}_{\sigma \gamma}(\hat{\mathbf{Y}}), \\
i\left[\hat{\hat{Q}}_{\alpha \beta}(\hat{\mathbf{Y}}), \hat{\hat{Q}}_{\gamma \rho}(\hat{\mathbf{Y}})\right] & \equiv-\frac{\hat{\hat{S}}_{\lambda}(\hat{\mathbf{Y}})}{4}\left(\delta_{\alpha \gamma} \varepsilon_{\beta \rho \lambda}+\delta_{\beta \gamma} \varepsilon_{\alpha \rho \lambda}+\delta_{\beta \rho} \varepsilon_{\alpha \gamma \lambda}+\delta_{\alpha \rho} \varepsilon_{\beta \gamma \lambda}\right) .
\end{aligned}
$$

Эти формулы нам потребуются для получения уравнений классификации вырожденных состояний равновесия.

\section{3. ВЫРОЖДЕННЫЕ СОСТОЯНИЯ РАВНОВЕСИЯ. ОСТАТОЧНАЯ СИММЕТРИЯ И УРАВНЕНИЯ КЛАССИФИКАЦИИ}

Для описания состояний равновесия со спонтанно нарушенной симметрией используем концепцию квазисредних [19], [20], [26]. Это значит, что статистический оператор Гиббса имеет вид

$$
\hat{\hat{w}}(Y, \xi)=e^{\Omega-Y_{a} \hat{\hat{\gamma}}_{a}-\nu \hat{\hat{F}}(\xi)} .
$$

Источник, который нарушает симметрию состояния равновесия, представляет собой линейный функционал оператора параметра порядка

$$
\hat{\hat{F}}=\int d^{3} x\left(f_{n}(\mathbf{x}, \xi) \hat{\hat{\Delta}}_{n}(\mathbf{x})+\text { э.c. }\right) .
$$

Здесь $f_{n}(\mathbf{x})$ - некоторая функция, сопряженная оператору параметра порядка, которая задает его равновесные значения $\Delta_{n}(\mathbf{x})=\left\langle\hat{\hat{\Delta}}_{n}(\mathbf{x})\right\rangle$. Квазисреднее определяется формулой

$$
\left\langle\hat{\hat{a}}_{n}(\mathbf{x})\right\rangle \equiv \lim _{\nu \rightarrow 0} \lim _{V \rightarrow 0} \operatorname{Sp} \hat{\hat{w}}(Y, \xi) \hat{\hat{a}}(\mathbf{x})
$$

где $\hat{\hat{a}}(\mathbf{x})$ - произвольный квазилокальный оператор. Выбор параметра порядка в выражении (6) связан с конкретной природой вырождения равновесных состояний конденсированных сред. Индекс $n$ характеризует тензорную структуру параметра порядка. Структура функций $f_{n}(\mathbf{x})$ определяется свойствами симметрии исследуемой фазы, что дает возможность ввести в рамках микроскопической теории в распределение Гиббса дополнительные термодинамические параметры $\xi$, которые в общем случае имеют тензорный характер. На конкретных примерах магнитных и сверхтекучих фазовых переходов мы показываем далее, как возникают дополнительные термодинамические параметры, которые характеризуют вырожденные состояния равновесия.

Приведем общую схему получения уравнений классификации для равновесных значений параметра порядка. Для этого нам понадобятся трансформационные свойства оператора параметра порядка. Генератором группы фазовых преобразований является оператор числа частиц $\hat{\hat{N}}$. Оператор параметра порядка $\hat{\hat{\Delta}}_{n}(\mathbf{x})$ преобразуется согласно соотношению

$$
\left[\hat{\hat{N}}, \hat{\hat{\Delta}}_{n}(\mathbf{x})\right]=-g \hat{\hat{\Delta}}_{n}(\mathbf{x})
$$


Постоянная $g$ зависит от тензорной размерности оператора параметра порядка. При преобразованиях, связанных с группой $S U(3)$-симметрии, генератором которой является оператор $\hat{\hat{G}}_{\alpha \beta}$, оператор параметра порядка $\hat{\hat{\Delta}}_{n}(\mathbf{x})$ преобразуется по представлениям этой группы:

$$
i\left[\hat{\hat{G}}_{\alpha \beta}, \hat{\hat{\Delta}}_{m}(\mathbf{x})\right]=-g_{\alpha \beta m n} \hat{\hat{\Delta}}_{n}(\mathbf{x})
$$

или в компактной записи $i\left[\hat{\hat{G}}_{\alpha \beta}, \hat{\hat{\Delta}}(\mathbf{x})\right]=-g_{\alpha \beta} \hat{\hat{\Delta}}(\mathbf{x})$, где $\left(\hat{g}_{\alpha \beta}\right)_{m n} \equiv g_{\alpha \beta m n}-$ постоянные матричные элементы, зависящие от тензорной размерности оператора параметра порядка. Из формулы (8) на основании тождества Якоби для операторов $\hat{\hat{G}}_{\alpha \beta}, \hat{\hat{G}}_{\mu \nu}$ и $\hat{\hat{\Delta}}_{n}(\mathbf{x})$ вытекает соотношение для матричных элементов

$$
i\left[\hat{g}_{\alpha \beta}, \hat{g}_{\mu \nu}\right]=\delta_{\mu \beta} \hat{g}_{\alpha \nu}-\hat{g}_{\mu \beta} \delta_{\alpha \nu} .
$$

Другим важным элементом статистической теории фазовых состояний является представление об остаточной симметрии состояния равновесия [11], [12]. Это значит, что статистический оператор вырожденных состояний равновесия удовлетворяет соотношению симметрии [22], [23], [26]

$$
[\hat{\hat{w}}(Y, \xi), \hat{\hat{T}}(\hat{\mathbf{Y}}, \xi)]=0 .
$$

Генератор остаточной симметрии $\hat{\hat{T}}$ является линейной комбинацией интегралов движения, по отношению к которым симметрия состояния равновесия нарушена. В случае спонтанного нарушения фазовой и магнитной симметрий состояния равновесия генератор остаточной симметрии имеет вид

$$
\hat{\hat{T}}(\hat{\mathbf{Y}}, \xi) \equiv \xi_{\alpha \beta} \hat{\hat{G}}_{\beta \alpha}(\hat{\mathbf{Y}})+c \hat{\hat{N}}=b_{\alpha} \hat{\hat{S}}_{\alpha}(\hat{\mathbf{Y}})+d_{\alpha \beta} \hat{\hat{Q}}_{\beta \alpha}(\hat{\mathbf{Y}})+c \hat{\hat{N}}
$$

В силу его эрмитовости параметры $\xi \equiv b_{\alpha}, d_{\alpha \beta}, c$ - действительные величины. Матрица $d_{\alpha \beta}$ является симметричной и бесследовой: $d_{\alpha \beta}=d_{\beta \alpha}, d_{\alpha \alpha}=0$. Пять ее независимых компонент мы параметризуем соотношением

$$
d_{\alpha \beta}=d_{1}\left(m_{\alpha} m_{\beta}-\frac{\delta_{\alpha \beta}}{3}\right)+d_{2}\left(n_{\alpha} n_{\beta}-\frac{\delta_{\alpha \beta}}{3}\right) .
$$

Здесь $d_{1}, d_{2}$ - модули этой матрицы. Векторы $m_{\alpha}, n_{\alpha}, l_{\alpha}=(\mathbf{m} \times \mathbf{n})_{\alpha}$ образуют ортонормированный репер. Разложение вектора $\mathbf{b}$ по этому реперу представим в виде $\mathbf{b}=b_{1} \mathbf{m}+b_{2} \mathbf{n}+b_{3} \mathbf{l}$. Наряду с термодинамическими силами $Y_{\alpha}$ параметры генератора остаточной симметрии $\xi \equiv b_{\alpha}, d_{\alpha \beta}, c$ представляют собой дополнительные термодинамические величины, которые характеризуют вырожденное состояние равновесия. Как мы увидим далее, в общем случае эти величины не являются независимыми. Условие остаточной симметрии (9) позволяет получить для параметра порядка линейные дифференциальные или, в частном случае, алгебраические уравнения, решения которых классифицируют вырожденные состояния равновесия в предположении его ненулевого равновесного значения в терминах параметров генератора остаточной симметрии. Допустимые значения коэффициентов генератора остаточной симметрии могут оказаться непрерывными величинами (например, если рассматривать оси магнитной анизотропии), а также иметь дискретный характер. 
Последние величины трактуются как квантовые числа или функции, которые различают квантовые состояния. Из условия остаточной симметрии (9) и трансформационных соотношений $(7),(8)$ приходим к линейным однородным дифференциальным уравнениям классификации равновесных значений параметра порядка

$$
\begin{gathered}
T_{m n}(\hat{\mathbf{Y}}, \xi) \Delta_{n}=0 \\
T_{m n}(\hat{\mathbf{Y}}, \xi) \equiv \xi_{\alpha \beta} g_{\beta \alpha m n}+i \delta_{m n}(c+\operatorname{tr} \hat{\xi} \hat{G}(\hat{\mathbf{Y}})) .
\end{gathered}
$$

Уравнения (11) упрощаются при $\hat{\mathbf{Y}}=0$ и становятся алгебраическими. Условие существования нетривиального решения для параметра порядка в последнем случае приобретает вид $\operatorname{det} \hat{T}(0, \xi)=0$. Далее мы используем представленную схему классификации для анализа магнитных и сверхтекучих состояний равновесия магнетиков со спином $s=1$, обладающих $S U(3)$-симметрией обменного взаимодействия.

\section{4. ЯВНЫЙ ВИД МАГНИТНЫХ ПАРАМЕТРОВ ПОРЯДКА В СОСТОЯНИИ РАВНОВЕСИЯ}

Рассмотрим частный случай вырожденных состояний равновесия, в которых нарушена только магнитная симметрии (4). В магнетиках с несколькими подрешетками генератор $S U(3)$-симметрии системы и магнитный оператор параметра порядка введем соотношениями

$$
\hat{\hat{G}}_{\alpha \beta} \equiv \int d^{3} x \sum_{n} \hat{\hat{g}}_{\alpha \beta}^{(n)}(\mathbf{x}), \quad \hat{\hat{\Delta}}_{\alpha \beta}(\mathbf{x}) \equiv \sum_{n} \theta_{n} \hat{\hat{g}}_{\alpha \beta}^{(n)}(\mathbf{x}),
$$

где операторы $n$-подрешетки $\hat{\hat{g}}_{\alpha \beta}^{(n)}(\mathbf{x})$ определяются выражением, аналогичным формуле $(2)$, и $\theta_{n}$ - некоторые произвольные постоянные, не обращающиеся в нуль или единицу одновременно. Операторы параметра порядка спинового нематика и антиферромагнетика связаны с оператором параметра порядка (12) равенством

$$
\hat{\hat{\Delta}}_{\alpha \beta}(\mathbf{x}) \equiv \hat{\hat{\Delta}}_{\alpha \beta}^{(s)}(\mathbf{x})+i \varepsilon_{\alpha \beta \gamma} \frac{\hat{\hat{\Delta}}_{\gamma}(\mathbf{x})}{2} .
$$

Принимая во внимание формулы (12), (13), получаем набор квантовых скобок Пуассона для этих операторов параметров порядка с магнитными интегралами движения

$$
\begin{aligned}
& i\left[\hat{\hat{S}}_{\alpha}, \hat{\hat{\Delta}}_{\beta}(\mathbf{x})\right]=-\varepsilon_{\alpha \beta \gamma} \hat{\hat{\Delta}}_{\gamma}(\mathbf{x}), \\
& i\left[\hat{\hat{S}}_{\alpha}, \hat{\hat{\Delta}}_{\mu \nu}^{(s)}(\mathbf{x})\right]=\varepsilon_{\alpha \sigma \mu} \hat{\hat{\Delta}}_{\sigma \nu}^{(s)}(\mathbf{x})+\varepsilon_{\alpha \sigma \nu} \hat{\hat{\Delta}}_{\sigma \mu}^{(s)}(\mathbf{x}), \\
& i\left[\hat{\hat{Q}}_{\alpha \beta}, \hat{\hat{\Delta}}_{\gamma}(\mathbf{x})\right]=-\varepsilon_{\alpha \gamma \sigma} \hat{\hat{\Delta}}_{\sigma \beta}(\mathbf{x})-\varepsilon_{\beta \gamma \sigma} \hat{\hat{\Delta}}_{\sigma \alpha}(\mathbf{x}), \\
& i\left[\hat{\hat{Q}}_{\alpha \beta}, \hat{\hat{\Delta}}_{\mu \nu}^{(s)}(\mathbf{x})\right]=\frac{\hat{\hat{\Delta}}_{\sigma}(\mathbf{x})}{4}\left(\varepsilon_{\mu \beta \sigma} \delta_{\alpha \nu}+\varepsilon_{\mu \alpha \sigma} \delta_{\beta \nu}+\varepsilon_{\nu \beta \sigma} \delta_{\alpha \mu}+\varepsilon_{\nu \alpha \sigma} \delta_{\beta \mu}\right) .
\end{aligned}
$$

Используя свойство остаточной симметрии (9) и учитывая соотношения (14), получаем при $\hat{\mathbf{Y}}=0$ систему линейных однородных алгебраических уравнений для обоих параметров порядка

$$
\begin{gathered}
\varepsilon_{\alpha \beta \gamma}\left(b_{\beta} \Delta_{\gamma}+2 d_{\beta \sigma} \Delta_{\gamma \sigma}^{(s)}\right)=0 \\
\varepsilon_{\alpha \beta \mu}\left(d_{\alpha \nu} \Delta_{\beta}-2 \Delta_{\alpha \nu}^{(s)} b_{\beta}\right)+\varepsilon_{\alpha \beta \nu}\left(d_{\alpha \mu} \Delta_{\beta}-2 \Delta_{\alpha \mu}^{(s)} b_{\beta}\right)=0 .
\end{gathered}
$$


Эти уравнения устанавливают их допустимые значения, если соответствующий детерминант матрицы обращается в нуль. Принимая во внимание явный вид (15), нетрудно видеть, что условие существования ненулевых решений параметров порядка справедливо при любых значениях вектора спонтанной анизотропии $\mathbf{b}$ и матрицы спонтанной анизотропии $\hat{d}$. Для нахождения искомых величин используем разложение антиферромагнитного параметра порядка по ортонормированному реперу $\boldsymbol{\Delta}=\Delta_{1} \mathbf{m}+\Delta_{2} \mathbf{n}+\Delta_{3} \mathbf{l}$. Аналогично, параметр порядка спинового нематика ищем в виде разложения $\Delta_{\alpha \beta}^{(s)}=\Delta_{n}^{(s)} F_{\alpha \beta}^{(n)}$ по полному набору симметричных и бесследовых матриц $F_{\alpha \beta}^{(n)}, n=1, \ldots, 5$, определенных равенствами

$$
\begin{gathered}
F_{\alpha \beta}^{(1)}=\frac{1}{3}\left(m_{\alpha} m_{\beta}-\delta_{\alpha \beta}\right), \quad F_{\alpha \beta}^{(2)}=\frac{1}{3}\left(n_{\alpha} n_{\beta}-\delta_{\alpha \beta}\right) \\
F_{\alpha \beta}^{(3)}=m_{\alpha} n_{\beta}+m_{\beta} n_{\alpha}, \quad F_{\alpha \beta}^{(4)}=m_{\alpha} l_{\beta}+m_{\beta} l_{\alpha}, \quad F_{\alpha \beta}^{(5)}=n_{\alpha} l_{\beta}+n_{\beta} l_{\alpha} .
\end{gathered}
$$

Уравнения (15) связывают действительные амплитуды антиферромагнитного $\Delta_{k}$, $k=1,2,3$, и тензорного параметров порядка $\Delta_{n}^{(s)}, n=1, \ldots, 5$. Получим теперь явный вид обоих параметров порядка для параметров спонтанной анизотропии $\mathbf{b}$ и $\hat{d}$. Эта ситуация является ситуацией общего положения, соответствующей трехосной анизотропии в спиновом пространстве. Из уравнений (15) найдем явный вид параметров порядка:

$$
\begin{gathered}
\Delta=b_{1} A_{+} \mathbf{m}+b_{2} A_{-} \mathbf{n}+b_{3} C \mathbf{l} \\
\Delta_{\alpha \beta}^{(s)}=\Delta_{*} \frac{1}{3}\left(b_{\alpha} b_{\beta}-b^{2} \delta_{\alpha \beta}\right)+\frac{d_{1} A_{-} F_{\alpha \beta}^{(1)}}{2}+\frac{d_{2} A_{+} F_{\alpha \beta}^{(2)}}{2} .
\end{gathered}
$$

Видим, что все восемь амплитуд обоих параметров порядка представимы в терминах двух независимых амплитуд $\Delta_{0}, \Delta_{*}$ :

$$
A_{ \pm} \equiv \Delta_{0}\left[1+\frac{d_{1}+d_{2}}{2}\right] \pm \Delta_{*}\left(d_{1}-d_{2}\right), \quad C \equiv \frac{A_{+}+A_{-}}{2}-\Delta_{*}\left(d_{1}+d_{2}\right)
$$

В частности, при $\mathbf{b}=0$ и $\hat{d}=d_{1} \hat{F}^{(1)}$ из выражений (17) получаем решения $\boldsymbol{\Delta}$ и $\Delta_{\alpha \beta}^{(s)}=\Delta_{\|}\left(e_{\alpha} e_{\beta}-\delta_{\alpha \beta} / 3\right)$ при $e_{\alpha} \| m_{\alpha}$ (случай спинового нематика типа легкая ось) и $\Delta_{\alpha \beta}^{(s)}=\Delta_{\perp}\left(e_{\alpha} e_{\beta}-\delta_{\alpha \beta} / 3\right)$ при $e_{\alpha} \perp m_{\alpha}$ (случай спинового нематика типа легкая плоскость). Принимая во внимание соотношения связи вектора магнитной анизотропии $\mathbf{b}$ с углами $\varphi$ и $\theta: b_{1}=b \cos \theta \cos \varphi, b_{2}=b \cos \theta \sin \varphi, b_{3}=b \sin \theta$, представим модуль антиферромагнитного параметра порядка в виде

$$
\Delta(\theta, \varphi)=b \sqrt{\cos ^{2} \theta\left(A_{+}^{2} \cos ^{2} \varphi+A_{-}^{2} \sin ^{2} \varphi\right)+C^{2} \sin ^{2} \theta}
$$

Не нарушая общности рассмотрения, положим величины $b=\Delta_{0}=1$ и приведем вид этой поверхности в сферической системе координат (см. рис. 1а-1г). Как видно из представленных рис. 1a, 1б, в одноосном случае матрицы $\hat{d}$ поверхность изменяется только вдоль оси 1. В двухосном случае матрицы $\hat{d}$ (см. рис. 1в, 1г) поверхность меняется в двух направлениях вдоль осей $\mathbf{m}$ и n. Нетрудно показать, что 
$\Delta_{n}=1$

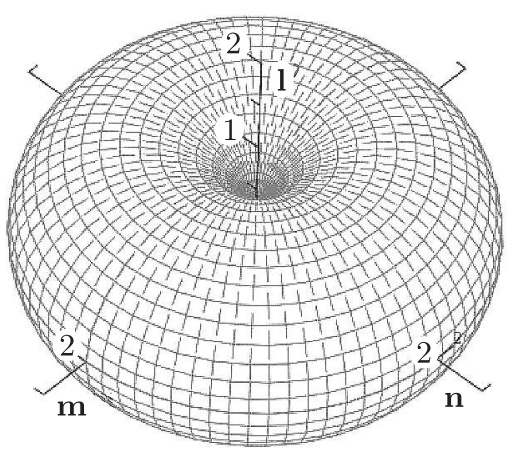

a

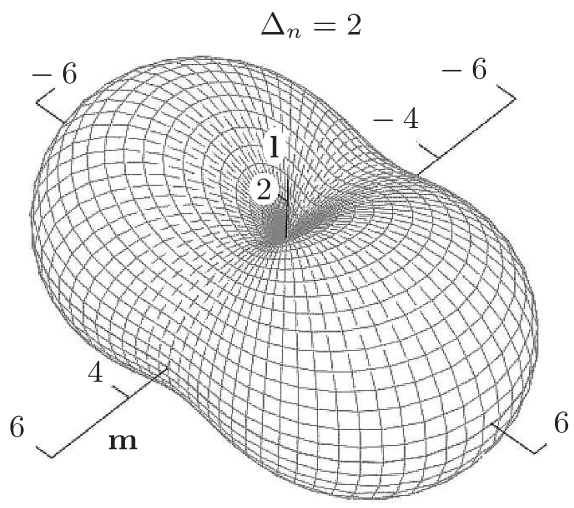

B

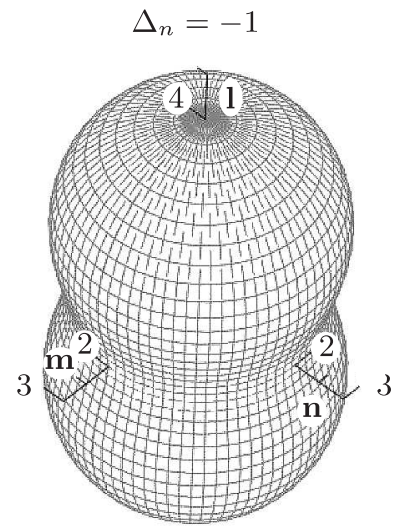

б

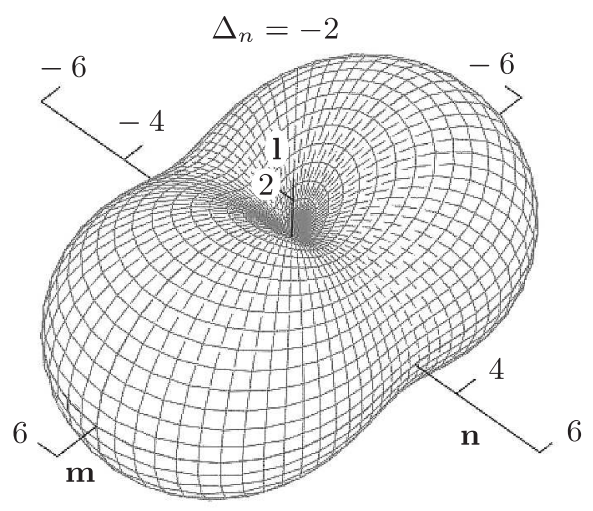

$\Gamma$

Рис. 1. Модуль антиферромагнитного параметра порядка при $b=\Delta_{0}=1$ и различных значениях $\Delta_{n}$.

угловая зависимость другого скалярного инварианта $\operatorname{tr}\left(\hat{\Delta}^{(s)}\right)^{2}$, связанного с параметром порядка спинового нематика, имеет аналогичный угловой вид, приведенный на рис. 1a-1г для модуля антиферромагнитного параметра порядка.

\section{5. АНИЗОТРОПИЯ ПАРАМЕТРА ПОРЯДКА В СВЕРХТЕКУЧИХ СОСТОЯНИЯХ РАВНОВЕСИЯ}

В этом разделе мы рассматриваем состояния равновесия, где одновременно спонтанно нарушены фазовая и магнитная симметрии. Прежде чем пояснить структуру оператора параметра порядка, характеризующего указанную нарушенную симметрию, сделаем следующее замечание. Адекватное описание сверхтекучего ${ }^{4} \mathrm{He}$ требует учета двух типов параметра порядка. Один из них линеен по полевому бозе-оператору $\hat{\Delta}(\mathbf{x})=\hat{\psi}(\mathbf{x})$ и введен в теорию в работе [27]. С этим параметром порядка связана одночастичная плотность конденсата $n_{0}$. Другой оператор параметра порядка, билинейный по полевым операторам $\hat{\Delta}(\mathbf{x})=\hat{\psi}(\mathbf{x}) \hat{\psi}(\mathbf{x})$, предло-

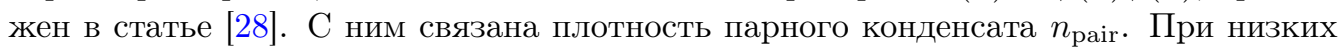


температурах для разреженных газов плотность парного конденсата очень мала, $n_{\text {pair }} / n_{0} \sim 10^{-6}[29]$. Для конденсированных состояний ситуация обратная - плотность парного конденсата доминирует, $n_{\text {pair }} / n_{0} \sim 10$ [30]-[32]. Поэтому при изучении сверхтекучих бозе-систем со спином $s \neq 0$ можно полагать, что билинейные операторы параметра порядка также важны для описания их физических свойств. Так как сверхтекучие состояния магнитных систем со спинорным параметром порядка $\hat{\Delta}_{\alpha}(\mathbf{x})=\hat{\psi}_{\alpha}(\mathbf{x})$ ранее рассмотрены в работах [8]-[10], [33], мы изучим иной характер нарушенной симметрии, который описывается оператором параметра порядка билинейным по полевым операторам (случай четного конденсата). С учетом магнитных степеней свободы оператор параметра порядка, отвечающий сверхтекучим состояниям бозе-системы со спином $s=1$, определим равенством $\hat{\Delta}_{\alpha \beta}(\mathbf{x})=\hat{\psi}_{\alpha}(\mathbf{x}) \hat{\psi}_{\beta}(\mathbf{x})$. Этот оператор симметричен, $\hat{\Delta}_{\alpha \beta}(\mathbf{x})=\hat{\Delta}_{\beta \alpha}(\mathbf{x})$, и для него имеет место квантовая алгебра

$$
\begin{aligned}
{\left[\hat{\hat{N}}, \hat{\hat{\Delta}}_{\alpha \beta}(\mathbf{x})\right]=- } & 2 \hat{\hat{\Delta}}_{\alpha \beta}(\mathbf{x}), \quad\left[\hat{\hat{S}}_{\gamma}, \hat{\hat{\Delta}}_{\alpha \beta}(\mathbf{x})\right]=i \varepsilon_{\gamma \alpha \rho} \hat{\hat{\Delta}}_{\rho \beta}(\mathbf{x})+i \varepsilon_{\gamma \beta \rho} \hat{\hat{\Delta}}_{\rho \alpha}(\mathbf{x}), \\
{\left[\hat{\hat{Q}}_{\mu \nu}, \hat{\hat{\Delta}}_{\alpha \beta}(\mathbf{x})\right]=} & -\frac{1}{2}\left(\delta_{\alpha \mu} \hat{\hat{\Delta}}_{\beta \nu}(\mathbf{x})+\delta_{\mu \beta} \hat{\hat{\Delta}}_{\alpha \nu}(\mathbf{x})+\delta_{\alpha \nu} \hat{\hat{\Delta}}_{\beta \mu}(\mathbf{x})+\delta_{\nu \beta} \hat{\hat{\Delta}}_{\alpha \mu}(\mathbf{x})\right)+ \\
& +\frac{2}{3} \delta_{\mu \nu} \hat{\hat{\Delta}}_{\alpha \beta}(\mathbf{x}) .
\end{aligned}
$$

Далее, следуя изложенному выше подходу, принимая во внимание формулы (18) и вид генератора остаточной симметрии (10), получаем уравнение классификации

$$
2 c \hat{\Delta}-i(\hat{\varepsilon}(\mathbf{b}) \hat{\Delta}+\hat{\Delta} \hat{\varepsilon}(\mathbf{b}))+\hat{d} \hat{\Delta}+\hat{\Delta} \hat{d}=0
$$

Здесь введено обозначение $\varepsilon_{\alpha \beta}(\mathbf{b}) \equiv \varepsilon_{\alpha \beta \gamma} b_{\gamma}$. Тензорный параметр порядка ищем в виде разложения по полному набору симметричных матриц:

$$
\Delta_{\alpha \beta}=\Delta_{(0)} \delta_{\alpha \beta}+\Delta_{(1)} F_{\alpha \beta}^{(1)}+\Delta_{(2)} F_{\alpha \beta}^{(2)}+\Delta_{(3)} F_{\alpha \beta}^{(3)}+\Delta_{(4)} F_{\alpha \beta}^{(4)}+\Delta_{(5)} F_{\alpha \beta}^{(5)} .
$$

Здесь $\Delta_{(n)}$ - комплексные амплитуды. Согласно выражению (19) допустимые значения для параметра $c$ получаем из уравнения

$$
\operatorname{det} \hat{D}=\left(c^{3}+p_{1} c+q_{1}\right)\left(c^{3}+\frac{p_{1} c}{4}-\frac{q_{1}}{8}\right)=0 .
$$

Входящие в него величины $p_{1}$ и $q_{1}$ определяются формулами

$$
\begin{aligned}
p_{1} & =-\frac{1}{2}\left(2+D_{2}\right)<0 \\
q_{1} & =-\cos ^{2} \theta\left(d_{1} \cos ^{2} \varphi+d_{2} \sin ^{2} \varphi\right)+\frac{1}{3}\left(d_{1}+d_{2}+D_{3}\right), \\
D_{2} & \equiv \operatorname{tr}(\hat{d})^{2}=\frac{2}{3}\left(d_{1}^{2}+d_{2}^{2}-d_{1} d_{2}\right)>0, \\
D_{3} & \equiv \operatorname{tr}(\hat{d})^{3}=\frac{1}{9}\left(d_{1}+d_{2}\right)\left(2 d_{1}-d_{2}\right)\left(d_{1}-2 d_{2}\right) .
\end{aligned}
$$


Из условия (20) находим шесть значений $c_{\mu}, \mu=1, \ldots, 6$, при которых $\left\langle\hat{\Delta}_{\alpha \beta}(\mathbf{x})\right\rangle \neq 0$, в виде функции скалярных инвариантов этого генератора:

$$
\begin{aligned}
& c_{1}=2 \sqrt[3]{R_{1}} \cos \frac{\chi_{1}}{3}, \\
& c_{2}=2 \sqrt[3]{R_{1}} \cos \frac{\chi_{1}+2 \pi}{3}, \\
& c_{3}=2 \sqrt[3]{R_{1}} \cos \frac{\chi_{1}+4 \pi}{3}, \quad c_{4}=2 \sqrt[3]{R_{2}} \cos \frac{\chi_{2}}{3}, \\
& c_{5}=2 \sqrt[3]{R_{2}} \cos \frac{\chi_{2}+2 \pi}{3}, \quad c_{6}=2 \sqrt[3]{R_{2}} \cos \frac{\chi_{2}+4 \pi}{3},
\end{aligned}
$$

где $R_{1} \equiv \sqrt{-p_{1}^{3} / 27}, \cos \chi_{1} \equiv-q_{1} / 2 R_{1}=-\cos \chi_{2}$ и $R_{2} \equiv R_{1} / 8$. Рассмотрим частные случаи параметров генератора магнитной анизотропии, для которых получим дискретные значения параметра $c_{\mu}$ и найдем явный вид равновесных значений тензорного параметра порядка. В силу уравнения (19) возникают связи амплитуд $\Delta_{(n)}$.

СлучАй 1 (b = bl, $\hat{d}=0$, одноосная остаточная симметрия). Тензорный параметр порядка и соответствующие значения $c_{\mu}$ имеют вид

$$
c_{1,2}=0, \quad \hat{\Delta}_{1,2}=\Delta_{(0)} \hat{I}+\Delta_{(1)}\left(\hat{F}^{(1)}+\hat{F}^{(2)}\right) .
$$

Структура этого параметра порядка в равновесии совпадает с решением, ранее найденным в работе [34], и соответствует "реальной" ("real") фазе. Другие два решения

$$
c_{3,4}= \pm b, \quad \hat{\Delta}_{3,4}=\Delta_{(1)}\left(\hat{F}^{(1)}-\hat{F}^{(2)} \mp i \hat{F}^{(3)}\right),
$$

совпадают с решением, полученным в работе [34], и отвечают “аксиальной” ("axial”) фазе; наконец,

$$
c_{5,6}= \pm \frac{b}{2}, \quad \hat{\Delta}_{5,6}=\Delta_{(4)}\left(\hat{F}^{(4)} \mp i \hat{F}^{(5)}\right) .
$$

Для разреженных бозе-систем обменные взаимодействия имеют $S O(3)$-симметрию [15] и генератор остаточной симметрии является функцией только оси магнитной анизотропии b. Приведенные далее примеры отвечают значениям $\hat{d} \neq 0$.

СлучАй $2\left(b_{\alpha}=0, \hat{d}=d_{1} \hat{F}^{(1)}\right.$, одноосная остаточная симметрия). Приведем дискретные значения параметра $c_{\mu}$ и соответствующий вид параметра порядка:

$$
\begin{aligned}
c_{1} & =-\frac{2 d_{1}}{3}, & \hat{\Delta}_{1} & =\Delta_{(1)}\left(\frac{\hat{I}}{3}+\hat{F}^{(1)}\right), \\
c_{2,3,4} & =\frac{d_{1}}{3}, & \hat{\Delta}_{2,3,4} & =\left(\Delta_{(2)}-2 \Delta_{(1)}\right) \frac{\hat{I}}{3}+\Delta_{(1)} \hat{F}^{(1)}+\Delta_{(2)} \hat{F}^{(2)}+\Delta_{(5)} \hat{F}^{(5)}, \\
c_{5,6} & =-\frac{d_{1}}{6}, & \hat{\Delta}_{5,6} & =\Delta_{(3)} \hat{F}^{(3)}+\Delta_{(4)} \hat{F}^{(4)} .
\end{aligned}
$$

СлучАй $3\left(b_{\alpha}=0, \hat{d}=d_{1} \hat{F}^{(1)}+d_{2} \hat{F}^{(2)}\right.$, двухосная остаточная симметрия). Собственные значения $c_{\mu}$ и параметр порядка принимают вид

$$
c_{1}=\frac{d_{2}-2 d_{1}}{3}, \quad \hat{\Delta}_{1}=\Delta_{(1)}\left(\frac{\hat{I}}{3}+\hat{F}^{(1)}\right), \quad 2 d_{1} \neq d_{2}, \quad d_{1} \neq d_{2} .
$$

Как мы видим из представленного решения, наличие двух параметров - модулей матрицы $\hat{d}$ - ведет к наличию дополнительных решений параметра порядка. Если 
$d_{1}=d_{1}$, то мы получаем решение, аналогичное рассмотренному в случае 2 . Выпишем решения для оставшихся собственных значений:

$$
\begin{aligned}
& c_{2}=\frac{d_{1}-2 d_{2}}{3}, \quad \hat{\Delta}_{2}=\Delta_{(2)}\left(\frac{\hat{I}}{3}+\hat{F}^{(2)}\right), \quad d_{1} \neq 2 d_{2}, \quad d_{1} \neq d_{2}, \\
& c_{3}=\frac{d_{1}+d_{2}}{3}, \quad \hat{\Delta}_{3}=\Delta_{(1)}\left(-\frac{\hat{I}}{3}+\hat{F}^{(1)}+\hat{F}^{(2)}\right), \quad d_{1} \neq \pm d_{2}, \\
& c_{4}=-\frac{d_{1}+d_{2}}{6}, \quad \hat{\Delta}_{4}=\Delta_{(3)} \hat{F}^{(3)}, \quad d_{1} \neq \pm d_{2}, \\
& c_{5}=\frac{2 d_{2}-d_{1}}{6}, \quad \hat{\Delta}_{5}=\Delta_{(4)} \hat{F}^{(4)}, \quad d_{1} \neq 2 d_{2}, \quad d_{1} \neq d_{2}, \\
& c_{6}=\frac{2 d_{1}-d_{2}}{6}, \quad \hat{\Delta}_{6}=\Delta_{(5)} \hat{F}^{(5)}, \quad 2 d_{1} \neq d_{2}, \quad d_{1} \neq d_{2} \text {. }
\end{aligned}
$$

СлУчАй 4 ( $\mathbf{b}=b_{1} \mathbf{m}, \hat{d}=d_{1} \hat{F}^{(1)}$, одноосная остаточная симметрия). Для дискретных значений параметра $c_{\mu}$ и параметра порядка получаем выражения

$$
\begin{array}{rlrl}
c_{1} & =-\frac{2 d_{1}}{3}, & \hat{\Delta}_{1}=\Delta_{(1)}\left(\frac{\hat{I}}{3}+\hat{F}^{(1)}\right), & b_{1} \neq \pm d_{1}, \\
c_{2,3}= \pm b_{1}+\frac{d_{1}}{3}, & \hat{\Delta}_{2,3}=\Delta_{(1)}\left(\hat{F}^{(1)}+2 \hat{F}^{(2)} \mp i \hat{F}^{(5)}\right), & b_{1} \neq \mp d_{1}, & 3 b_{1} \neq \mp d_{1}, \\
c_{4} & =\frac{d_{1}}{3}, & \hat{\Delta}_{4}=\Delta_{(1)}\left(-\frac{2 \hat{I}}{3}+\hat{F}^{(1)}\right), & \\
c_{5,6}= \pm b_{1}-\frac{d_{1}}{6}, & \hat{\Delta}_{5,6}=d_{1}, &
\end{array}
$$

СлУчАЙ $5\left(\mathbf{b}=b_{1} \mathbf{n}, \hat{d}=d_{1} \hat{F}^{(1)}\right.$, двухосная остаточная симметрия). Значения $c_{\mu}$ и выражения для тензорного параметра порядка имеют вид

$$
\begin{gathered}
c_{1}=\frac{d_{1}}{3}, \quad \hat{\Delta}_{1}=\Delta_{(2)}\left(\frac{\hat{I}}{3}+\hat{F}^{(2)}\right), \\
c_{2,3}=-\frac{d_{1} \pm 3 \sqrt{d_{1}^{2}+4 b^{2}}}{6} \\
\hat{\Delta}_{2,3}=\Delta_{(1)}\left(-d_{1} \frac{\hat{I}}{3}+\sqrt{d_{1}^{2}+4 b^{2}} \hat{F}^{(1)}+\left( \pm d_{1}+\sqrt{d_{1}^{2}+4 b^{2}}\right) \frac{\hat{F}^{(2)}}{2} \pm i b \hat{F}^{(4)}\right), \\
c_{4}=-\frac{d_{1}}{6}, \quad \hat{\Delta}_{4}=\Delta_{(2)}\left(-\frac{4 b \hat{I}}{3}+2 b \hat{F}^{(2)}-i d_{1} \hat{F}^{(4)}\right) \\
c_{5,6}=\frac{d_{1} \pm 3 \sqrt{d_{1}^{2}+4 b^{2}}}{12}, \quad \hat{\Delta}_{5,6}=\Delta_{(3)}\left(2 b \hat{F}^{(3)}+i\left(d_{1} \pm \sqrt{d_{1}^{2}+4 b^{2}}\right) \hat{F}^{(5)}\right) .
\end{gathered}
$$

Представленные формулы демонстрируют разнообразие сверхтекучих состояний равновесия, описываемых тензорным параметром порядка, которые связаны с появлением дополнительной термодинамической величины - матрицы $\hat{d}$. Эта термодинамическая величина возникает в магнитных системах благодаря $S U(3)$-симметрии обменного взаимодействия. Реализация таких магнитных и сверхтекучих состояний возможна для достаточно плотных состояний бозе-систем (жидкостей, твердых тел). 
Всем допустимым значениям величины $c_{\mu}$, которые следуют из уравнения классификации, отвечает равновесная структура параметра порядка с амплитудами $\Delta_{(n)}$. Отчасти приведенный вид сверхтекучего параметра порядка и параметра порядка спинового нематика напоминает структуру решений параметра порядка жидких кристаллов в описании нематических жидких кристаллов типа "twist-bend" [35].

Сравним структуру генератора остаточной симметрии для приведенных сверхтекучих состояний равновесия с генератором остаточной симметрии для состояний равновесия сверхтекучего ${ }^{3}$ Не. Четыре типа генераторов остаточной симметрии сверхтекучих фаз ${ }^{3} \mathrm{He}[26]$, [36] можно охарактеризовать с помощью двух квантовых чисел $m_{l}=0, \pm 1$ и $m_{s}=0, \pm 1$. Для рассмотренной сверхтекучей бозе-системы со спином $s=1$ шесть величин $c_{\mu}$ представляют собой квантовые функции, которые построены из скалярных инвариантов параметров магнитной анизотропии генератора остаточной симметрии.

\section{6. НЕОДНОРОДНЫЕ МАГНИТНЫЕ СОСТОЯНИЯ РАВНОВЕСИЯ}

Рассмотрим вопрос классификации магнитных состояний равновесия в пространственно неоднородном случае, при котором одновременно нарушены соотношение симметрии в спиновом пространстве (4) и условие пространственной однородности (3). В рамках феноменологической теории неоднородные состояния равновесия магнетиков со спином $s=1 / 2$ ранее изучены в работе [37], где учитывалась $S O(3)$-симметрия обменного взаимодействия гамильтониана. Вопрос классификации неоднородных магнитных состояний с этим же значением спина рассмотрен в статьях [23], [38]. Аналогично представлению об остаточной симметрии состояния равновесия, для магнетиков со спином $s=1$ рассмотрим генератор трансляционной симметрии вида $\hat{\hat{P}}_{k}(\eta) \equiv \hat{\hat{P}}_{k}-q_{k, \alpha} \hat{\hat{S}}_{\alpha}-d_{k, \alpha \beta} \hat{\hat{Q}}_{\alpha \beta}$, который включает в свое определение наряду с оператором импульса также магнитные интегралы движения. Постоянные величины $\eta=q_{k, \alpha}, d_{k, \alpha \beta}$ представляют собой дополнительные термодинамические параметры, которые характеризуют пространственную неоднородность и магнитную анизотропию состояния равновесия. Тензор $d_{k, \alpha \beta}$ симметричен и не имеет следа: $d_{k, \alpha \beta}=d_{k, \beta \alpha}, d_{k, \alpha \alpha}=0$. Далее мы исследуем случай, когда генератор трансляционной симметрии удовлетворяет стандартному соотношению коммутации для компонент оператора импульса $\left[\hat{\hat{P}}_{k}(\eta), \hat{\hat{P}}_{i}(\eta)\right]=0$. Это требование с учетом явного вида генератора трансляционной симметрии и квантовой алгебры (5) приводит к равенствам

$$
q_{i, \alpha} q_{k, \beta} \varepsilon_{\alpha \beta \gamma}+\left(\hat{d}_{k} \hat{d}_{i}\right)_{\beta \alpha} \varepsilon_{\alpha \beta \gamma}=0, \quad q_{i} d_{k, \gamma \rho}-q_{k, \alpha} d_{i, \gamma \rho}=0,
$$

которые упрощают структуру этих термодинамических параметров. Легко видеть, что представление этих величин в виде $q_{k, \alpha}=q_{k} f_{\alpha}$ и $d_{k, \alpha \beta}=d_{k} f_{\alpha \beta}$ удовлетворяет соотношениям (21), если $q_{k} \| d_{k}$. Далее мы полагаем, что $q_{k}=d_{k}$. Таким образом, генератор трансляционной симметрии приобретает вид

$$
\hat{\hat{P}}_{k}(\eta) \equiv \hat{\hat{P}}_{k}-q_{k}\left(f_{\alpha} \hat{\hat{S}}_{\alpha}+f_{\alpha \beta} \hat{\hat{Q}}_{\alpha \beta}\right) .
$$

Величина $q_{k}$ есть вектор магнитной спирали, $2 \pi / q-$ шаг спирали, $f_{\alpha}-$ вектор спонтанной магнитной анизотропии и $f_{\alpha \beta}$ - матрица спонтанной магнитной анизотропии. 
Неоднородное состояние равновесия магнетиков со спином $s=1$ обладает трансляционной симметрией в смысле соотношения

$$
\left[\hat{\hat{w}}, \hat{\hat{P}}_{k}(\eta)\right]=0 .
$$

Используя тождество Якоби для операторов $\hat{\hat{w}}, \hat{\hat{T}}, \hat{\hat{P}}_{k}$, получаем следующее соотношение: $\left[\left[\hat{\hat{T}}, \hat{\hat{P}}_{k}\right], \hat{\hat{w}}\right]=0$, которое связывает параметры генераторов остаточной и трансляционной симметрии. Далее для простоты мы рассматриваем случай, при котором векторы $b_{\alpha}$ и $f_{\alpha}$, а также тензоры $f_{\alpha \beta}$ и $d_{\alpha \beta}$ совпадают, так что тождество Якоби выполняется тривиальным образом.

Принимая во внимание алгебру аддитивных интегралов движения и параметров порядка (13), (14), соотношение $i\left[\hat{\hat{P}}_{k}, \hat{\hat{\Delta}}_{\alpha \beta}(\mathbf{x})\right]=-\nabla_{k} \hat{\hat{\Delta}}_{\alpha \beta}(\mathbf{x})$, а также условие симметрии (23) и явный вид (22), получаем систему уравнений, которые устанавливают магнитную и пространственную структуру обоих параметров порядка:

$$
\begin{aligned}
\nabla_{i} \Delta_{\rho}(\mathbf{x}) & =q_{i}\left(f_{\alpha} \varepsilon_{\alpha \rho \sigma} \Delta_{\sigma}(\mathbf{x})+2 f_{\alpha \beta} \varepsilon_{\alpha \rho \delta} \Delta_{\delta \beta}^{(s)}(\mathbf{x})\right) \\
\nabla_{i} \Delta_{\mu \nu}^{(s)}(\mathbf{x}) & =q_{i} f_{\alpha}\left(\varepsilon_{\alpha \mu \sigma} \Delta_{\sigma \nu}^{(s)}(\mathbf{x})+\varepsilon_{\alpha \nu \sigma} \Delta_{\sigma \mu}^{(s)}(\mathbf{x})\right)-\frac{q_{i} \Delta_{\sigma}}{2}(\mathbf{x})\left(\varepsilon_{\sigma \mu \beta} f_{\nu \beta}+\varepsilon_{\sigma \nu \beta} f_{\mu \beta}\right) .
\end{aligned}
$$

1. Рассмотрим частный случай уравнений (24), при котором $f_{\alpha}=f m_{\alpha}, m_{\alpha}^{2}=1$ и $f_{\alpha \beta}=0$. Уравнения для параметров порядка разделяются,

$$
\begin{aligned}
\nabla_{i} \triangle_{\rho}(\mathbf{x}) & =q_{i} f_{\alpha} \varepsilon_{\alpha \rho \sigma} \Delta_{\sigma}(\mathbf{x}) \\
\nabla_{i} \triangle_{\mu \nu}^{(s)}(\mathbf{x}) & =q_{i} f_{\alpha}\left(\varepsilon_{\alpha \mu \sigma} \Delta_{\sigma \nu}^{(s)}(\mathbf{x})+\varepsilon_{\alpha \nu \sigma} \Delta_{\sigma \mu}^{(s)}(\mathbf{x})\right) .
\end{aligned}
$$

Решение для антиферромагнитного параметра порядка записывается как $\Delta_{\rho}(\mathbf{x})=$ $a_{\rho \lambda}(\mathbf{x}) \underline{\Delta}_{\lambda}(0)$, где зависимость от координаты несет в себе ортогональная матрица поворота

$$
\begin{gathered}
a_{\rho \lambda}(\mathbf{x})=a_{\rho \lambda}(\mathbf{m} \theta(\mathbf{x}))=\delta_{\rho \lambda} \cos \theta(\mathbf{x})+m_{\rho} m_{\lambda}(1-\cos \theta(\mathbf{x}))+\varepsilon_{\rho \lambda \sigma} m_{\sigma} \sin \theta(\mathbf{x}), \\
\theta(\mathbf{x})=\theta+f \mathbf{q} \mathbf{x} .
\end{gathered}
$$

Из второго уравнения в (25) получаем решение для параметра порядка спинового нематика $\Delta_{\mu \nu}^{(s)}(\mathbf{x})=a_{\mu \sigma}(\mathbf{x}) a_{\nu \delta}(\mathbf{x}) \Delta_{\sigma \delta}^{(s)}(0)$ с этой же ортогональной матрицей. Такое решение описывает модуляцию оси спиновой анизотропии параметра порядка спинового нематика.

2. Рассмотрим другой частный случай, при котором $f_{\alpha}=0$ и $f_{\alpha \beta} \neq 0$. Согласно формулам (24) записываем уравнения для установления координатной зависимости антиферромагнитного параметра порядка и параметра порядка спинового нематика

$$
\begin{aligned}
\nabla_{i} \Delta_{\rho}(\mathbf{x}) & =2 q_{i} f_{\alpha \beta} \varepsilon_{\alpha \rho \delta} \Delta_{\delta \beta}^{(s)}(\mathbf{x}), \\
\nabla_{i} \Delta_{\mu \nu}^{(s)}(\mathbf{x}) & =-\frac{q_{i} \Delta_{\sigma}(\mathbf{x})}{2}\left(\varepsilon_{\sigma \mu \beta} f_{\nu \beta}+\varepsilon_{\sigma \nu \beta} f_{\mu \beta}\right) .
\end{aligned}
$$

Отсюда следует замкнутое уравнение для параметра порядка спинового нематика

$$
\triangle \Delta_{\mu \nu}^{(s)}(\mathbf{x})=-q^{2}\left(\hat{f}^{2} \hat{\Delta}^{(s)}(\mathbf{x})+\hat{\Delta}^{(s)}(\mathbf{x}) \hat{f}^{2}-2 \hat{f} \hat{\Delta}^{(s)}(\mathbf{x}) \hat{f}\right)_{\mu \nu} .
$$


Здесь $\triangle \equiv \nabla_{\alpha} \nabla_{\alpha}$. Рассматривая для простоты одноосную структуру тензора магнитной анизотропии $f_{\alpha \beta} \equiv f\left(n_{\alpha} n_{\beta}-\delta_{\alpha \beta} / 3\right)$, приходим к уравнению

$$
\triangle_{\mu \nu}^{(s)}(\mathbf{x})=q^{2} f^{2}\left(2 n_{\mu} n_{\nu} n_{\alpha} \hat{\Delta}_{\alpha \beta}^{(s)}(\mathbf{x}) n_{\beta}-n_{\mu} n_{\sigma} \hat{\Delta}_{\sigma \nu}^{(s)}(\mathbf{x})-n_{\nu} n_{\sigma} \hat{\Delta}_{\sigma \mu}^{(s)}(\mathbf{x})\right) .
$$

Решение этого уравнения ищем в виде разложения параметра порядка спинового нематика в ряд по полному набору симметричных и бесследовых матриц, которые определены равенствами $(16): \hat{\Delta}(\mathbf{x})=A_{l}(\mathbf{x}) \hat{F}^{(1)}$, где $l=1, \ldots, 5$ и $A_{l}(\mathbf{x})$ - неизвестные амплитуды, являющиеся функциями координат. Согласно уравнению (28) получаем уравнение связи этих амплитуд $\triangle A_{l}(\mathbf{x}) \hat{F}^{(1)}=-q^{2} f^{2}\left(A_{3}(\mathbf{x}) \hat{F}^{(3)}+A_{5}(\mathbf{x}) \hat{F}^{(5)}\right)$. Если потребовать выполнения условия ограниченности решения на бесконечности, то отсюда следует координатная зависимость параметра порядка спинового нематика $\hat{\Delta}^{(s)}(\mathbf{x})=\underline{A}_{3} \cos \theta(\mathbf{x}) \hat{F}^{(3)}+\underline{A}_{5} \sin \theta(\mathbf{x}) \hat{F}^{(5)}$. Здесь фаза имеет вид $\theta(\mathbf{x})=2 f \mathbf{q} \mathbf{x}+\theta$. Аналогичным образом выводим и пространственную зависимость антиферромагнитного параметра порядка $\hat{\Delta}(\mathbf{x})=\underline{A}_{3} \sin \theta(\mathbf{x}) l_{\rho}+\underline{A}_{5} \cos \theta(\mathbf{x}) m_{\rho}$.

Представленный подход позволяет установить равновесную структуру антиферромагнитного параметра порядка и параметра порядка спинового нематика в общем виде. На основе приведенного анализа сверхтекучих состояний равновесия можно получить вид шести типов генератора ненарушенной симметрии

$$
\hat{\hat{T}}_{\mu} \equiv c_{\mu} \hat{\hat{N}}+b_{\alpha} \hat{\hat{S}}_{\alpha}+d_{\alpha \beta} \hat{\hat{Q}}_{\alpha \beta} \text {. }
$$

Наряду с осями анизотропии матрицы $\hat{d}$ и вектора $\mathbf{b}$ этот оператор также является функцией дискретной величины $c_{\mu}$, которая классифицирует вырожденные состояния равновесия при наличии магнитных степеней свободы. В отличие от сверхтекучих состояний ${ }^{3} \mathrm{He}$, которые характеризуются двумя квантовыми числами, рассмотренные сверхтекучие состояния бозе-системы со спином $s=1$ и $S U(3)$-симметрией обменного взаимодействия характеризуются шестью квантовыми скалярными функциями.

\section{Список литературы}

[1] C. Lacroix, P. Mendels, F. Mila (eds.), Introduction to Frustrated Magnetism: Materials, Experiments, Theory, Springer Series in Solid-State Sciences, 164, Springer, Berlin, Heidelberg, 2011.

[2] A. Lauchli, F. Mila, K. Penc, "Quadrupolar phases of the $S=1$ bilinear-biquadratic Heisenberg model on the triangular lattice", Phys. Rev. Lett., 97:8 (2006), 087205, 4 pp.; Erratum, 97:22 (2006), 229901.

[3] P. Li, G.-M. Zhang, S.-Q. Shen, " $S U(3)$ bosons and the spin nematic state on the spin-1 bilinear-biquadratic triangular lattice", Phys. Rev. B, 75:10 (2007), 104420, 8 pp.

[4] P. Santini, S. Carretta, G. Amoretti, R. Caciuffo, N. Magnani, G. H. Lander, "Multipolar interactions in $f$-electron systems: the paradigm of actinide dioxides", Rev. Modern Phys., 81:2 (2009), 807-863.

[5] M. E. Zhitomirsky, H. Tsunetsugu, "Magnon pairing in quantum spin nematic", Europhys. Lett., 92:3 (2010), 37001.

[6] V. G. Bar'yakhtar, V.I. Butrim, A. K. Kolezhuk, B. A. Ivanov, "Dynamics and relaxation in spin nematics", Phys. Rev. B, 87:22 (2013), 224407, 9 pp.

[7] T. Zibold, V. Corre, C. Frapolli, A. Invernizzi, J. Dalibard, F. Gerbier, "Spin-nematic order in antiferromagnetic spinor condensates", Phys. Rev. A, 93:2 (2016), 023614, 13 pp. 
[8] T.-L. Ho, "Spinor bose condensates in optical traps", Phys. Rev. Lett., 81:4 (1998), 742-745.

[9] T. Ohmi, T. Machida, "Bose-Einstein condensation with internal degrees of freedom in alkali atom gases", J. Phys. Soc. Japan, 67 (1998), 1822-1825.

[10] R. Barnett, A. Turner, E. Demler, "Classifying novel phases of spinor atoms", Phys. Rev. Lett., 97:18 (2006), 180412, 4 pp., arXiv: cond-mat/0607253.

[11] L. Michel, "Symmetry defects and broken symmetry. Configurations Hidden symmetry", Rev. Modern Phys., 52:3 (1980), 617-651.

[12] В.П. Минеев, Топологически устойчивые неоднородные состояния в упорядоченных средах, Препринт ИТФ им. Л. Д. Ландау, ИТФ, Черноголовка, 1980.

[13] H. Mäkela, K. A. Suominen, "Inert states of spin-S systems", Phys. Rev. Lett., 99:19 (2007), 190408, 4 pp.

[14] S.-K. Yip, "Symmetry and inert states of spin Bose-Einstein condensates", Phys. Rev. A, 75:2 (2007), 023625, 9 pp.

[15] Y. Kawaguchi, M. Ueda, "Spinor Bose-Einstein condensates", Phys. Rep., 520:5 (2012), 253-381.

[16] F. Zhou, M. Snoek, "Spin singlet Mott states and evidence for spin singlet quantum condensates of spin-one bosons in lattices", Ann. Phys., 308:2 (2003), 692-738.

[17] Y.-Q. Li, S.-J. Gu, Z.-J. Ying, "One-dimensional $S U(3)$ bosons with $\delta$-function interaction", J. Phys. A: Math. Gen., 36:11 (2003), 2821-2838.

[18] C. M. Puetter, M. J. Lawler, H.-Y. Kee, "Theory of the spin-nematic to spin-Peierls quantum phase transition in ultracold spin-1 atoms in optical lattices", Phys. Rev. B, 78:16 (2008), 165121, 6 pp.

[19] Н.Н. Боголюбов, Квазисредние в задачах статистической механики, Препринт Д-781, ОИЯИ, Дубна, 1961.

[20] Н. Н. Боголюбов, Н. Н. Боголюбов (мл.), Введение в квантовую статистическую механику, Наука, М., 1984.

[21] Н.Н.Боголюбов,(мл.), М. Ю. Ковалевский, А.М.Курбатов, С. В. Пелетминский, А.Н. Тарасов, "К микроскопической теории сверхтекучих жидкостей”, УФН, 159:4 (1989), 585-620.

[22] М. Ю. Ковалевский, С. В. Пелетминский, "Статистическая механика квантовых жидкостей с триплетным спариванием", ЭЧАЯ, 33:6 (2002), 1357-1442.

[23] N. N. Bogolyubov Jr., D. A. Demyanenko, M. Y. Kovalevsky, N. N. Chekanova, "Quasiaverages and classification of equilibrium states of condensed media with spontaneously broken symmetry", Phys. Atom. Nucl., 72:5 (2009), 761-767.

[24] М. Ю. Ковалевский, "О классификации магнитных и сверхтекучих состояний равновесия в магнетиках со спином $s=1 ", T M \Phi, 186$ (2016), 456-474.

[25] N. Papanicolaou, "Unusual phases in quantum spin-1 systems", Nucl. Phys. B, 305:3 (1988), 367-395.

[26] М. Ю. Ковалевский, С.В. Пелетминский, Статистическая механика квантовых жидкостей и кристаллов, Физматлит, М., 2006.

[27] Н.Н. Боголюбов, "К теории сверхтекучести", Изв. АН СССР. Сер. физ., 11 (1947), $77-90$.

[28] E. P. Gross, "Quantum theory of interacting bosons", Ann. Phys., 9 (1960), 292-324.

[29] A. S. Peletminskii, S. V. Peletminskii, Yu. M. Poluektov, "Role of single-particle and pair condensates in Bose systems with arbitrary intensity of interaction", Condens. Matter Phys., 16:1 (2013), 13603, 17 pp., arXiv: 1303.5539.

[30] И. В. Богоявленский, Л.В.Карнацевич, Ж. А. Козлов, А.В.Пучков, "Бозе-конденсация в жидком ${ }^{4} \mathrm{He} ", \Phi H T, \mathbf{1 6 : 2}$ (1990), 139-162.

[31] H. R. Glyde, S. O. Diallo, R. T. Azuah, O. Kirichek, J. W. Taylor, "Bose-Einstein condensation in liquid ${ }^{4}$ He under pressure", Phys. Rev. B, 83:10 (2011), 100507, 4 pp.

[32] И. А. Вакарчук, "Матрицы плотности сверхтекучего гелия-4. II", TMФ, 82:3 (1990), 438-449. 
[33] А. И. Ахиезер, С. В. Пелетминский, Ю. В. Слюсаренко, "К теории слабонеидеального бозе-газа в магнитном поле", ЖЭТФ, 113:3 (1998), 918-929.

[34] N. D. Mermin, " $d$-Wave pairing near the transition temperature", Phys. Rev. A, 9:2 (1974), $868-872$.

[35] E. I. Kats, "Spontaneous chiral symmetry breaking in liquid crystals", ФHT, 43:1 (2017), $7-10$.

[36] D. Vollhardt, P. Wölfle, The Superfluid Phases of Helium 3, Taylor and Francis, London, New York, 1990.

[37] И.Е. Дзялошинский, “Теория геликоидальных структур в антиферромагнетиках. І. Неметаллы”, ЖЭТФ, 46:4 (1964), 1420-1437.

[38] М. Ю. Ковалевский, "Квазисредние в решении задачи классификации состояний равновесия конденсированных сред со спонтанно нарушенной симметрией”, ТMФ, 160:2 (2009), 290-303.

Поступила в редакцию 3.05.2017, после доработки 30.05.2017 